\title{
Research on Control Technology of Permanent Magnet Synchronous Motor Based on Iterative Algorithm
}

\author{
Liu Yangyang ${ }^{1 \mathrm{c}}$, Yang Guang ${ }^{2 b}$, Zou Qi ${ }^{1 \mathrm{c}}$, \\ ${ }^{1}$ Electric Power Science \& Research Institute of State Grid Tianjin Electirc Power \\ Company, Tianjin,China, 300384 \\ ${ }^{2}$ State Grid Tianjin Electirc Power Company,Tianjin,China,300010 \\ aliuyangyangnhm@sina.com, ${ }^{b}$ capshine@126.com, 'czouqi1004@163.com
}

Keywords: permanent magnet synchronous motor; position detection; iterative method, simulation

\begin{abstract}
The position detection is the important link in the permanent magnet synchronous motor control. The accuracy of the detection is the key to the high performance control of the motor. Aiming at the problem of permanent magnet synchronous motor pole position difficult to detect, of permanent magnet synchronous motor position loop were in-depth analysis, and mathematical model as the basis, find out factors that can influence the precision of motor control, a design based on iterative algorithm for the permanent magnet synchronous motor position controller. The simulation results show that this method can improve the dynamic performance of the system and improve the tracking accuracy of the system, and it has some application value.
\end{abstract}

\section{Introduction}

High performance AC servo system is the basis of advanced manufacturing industry, which reflects the development level of a country's industry [1][2]. The permanent magnet synchronous motor has the advantages of high power, low noise, low rotational inertia, good control effect, etc., and has occupied the main position in AC servo system.

The structure of permanent magnet synchronous motor is complex and the magnetic pole position is difficult to detect. So, it is a hot spot to find an effective position control strategy for motor pole position [3][4]. The in the permanent magnet synchronous motor position control system as the research object, with duplication of operation characteristics of the position control system, the iterative algorithm is applied to the pole placement control strategy, has been gradually narrowing in the iterative process, the error. In the end, the servo system of the actual output and the target output consistent.

\section{Theoretical overview of permanent magnet synchronous motor}

Composition of permanent magnet synchronous motor. Common permanent magnet synchronous motor can be divided into driven sine wave and square wave driving. In this paper, the sine wave drive of permanent magnet synchronous motor as the research object, the structure includes two parts of the stator and rotor.

\section{Characteristics of permanent magnet synchronous motor}

The motor speed and power frequency always keep the relationship, the frequency of the control can control the motor speed.

Permanent magnet synchronous motor has good anti-interference ability, and the change of load is small.

Permanent magnet synchronous motor with permanent magnet on the rotor without excitation, so the motor can keep the synchronous operation at a very low speed, and speed range is wide.

Mathematical model of permanent magnet synchronous motor. The mathematical model is accurate, which directly determines the performance of PMSM [5]. Neglecting core saturation and hysteresis loss, the motor is simplified, as shown in Figure 1. 


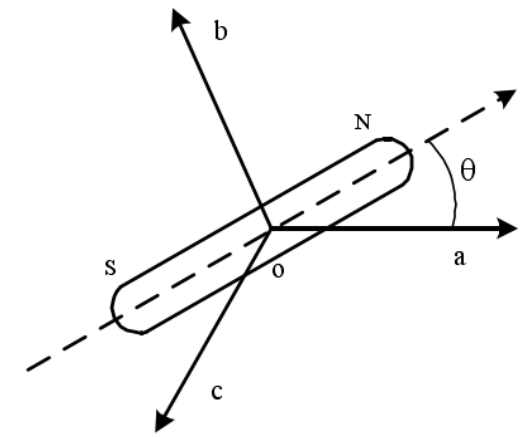

Fig.1 simplified model of permanent magnet synchronous motor

\section{Physical equation [6]}

$$
\left[\begin{array}{l}
u_{a} \\
u_{b} \\
u_{c}
\end{array}\right]=\left[\begin{array}{ccc}
R_{a} & 0 & 0 \\
0 & R_{b} & 0 \\
0 & 0 & R_{c}
\end{array}\right]\left[\begin{array}{l}
i_{a} \\
i_{b} \\
i_{c}
\end{array}\right]+\frac{d}{d t}\left[\begin{array}{l}
\varphi_{a} \\
\varphi_{b} \\
\varphi_{c}
\end{array}\right]
$$

Where:

$i_{a}, i_{b}, i_{c}$-Current of three-phase stator winding;

$u_{a}, u_{b}, u_{c}-$ Voltage of three-phase stator winding;

$\varphi_{a}, \varphi_{b}, \varphi_{c}$ - linkage of three-phase stator winding;

$R_{a}, R_{b}, R_{c}$-Resistance of three-phase stator winding.

\section{Linkage equation}

Where:

$$
\left[\begin{array}{l}
\varphi_{a} \\
\varphi_{b} \\
\varphi_{c}
\end{array}\right]=\left[\begin{array}{lll}
L_{a a}(\theta) & M_{a b}(\theta) & M_{a c}(\theta) \\
M_{b a}(\theta) & L_{b b}(\theta) & M_{b c}(\theta) \\
M_{c a}(\theta) & M_{c b}(\theta) & L_{c c}(\theta)
\end{array}\right]\left[\begin{array}{c}
i_{a} \\
i_{b} \\
i_{c}
\end{array}\right]+\left[\begin{array}{c}
\varphi_{f}{ }^{a}(\theta) \\
\varphi_{f}{ }^{b}(\theta) \\
\varphi_{f}{ }^{c}(\theta)
\end{array}\right]
$$

$\theta$-Rotor angle, $L_{X X}(\theta)$ - Self-inductance of each phase winding;

$M_{X X}(\theta)$-Mutual inductance of each phase winding;

\section{Electromagnetic torque equation}

$$
T_{e}=-p_{n} \varphi_{f}\left[i_{a} \sin \theta+i_{b} \sin \left(\theta-\frac{2}{3} \pi\right)+i_{c} \sin \left(\theta-\frac{4}{3} \pi\right)\right]
$$

Where:

$p_{n}$-Number of pole-pairs,

\section{Motion equation}

$$
J \frac{d \omega_{r}}{d t}=T_{e}-T_{L}-B \omega_{r}
$$

Where:

$J$-Moment of inertia; $\omega_{r}$-Angular velocity of the rotor;

$T_{L}$ - Load torque; $B$ - viscous friction coefficient.

\section{Position loop analysis of permanent magnet synchronous motor}

Permanent magnet synchronous motor position control system is by the position loop, speed loop and current loop composition. Current loop is the permanent magnet synchronous motor position control system constitutes the fundamental, and the dynamic characteristics are related to the realization of vector control strategy. Speed loop is also an extremely important link, which can quickly and accurately track the position loop of the output. The design of position loop should pay 
attention to the precision of fast tracking and position tracking, so as to guarantee the performance of the system[7][8].

The position loop is a permanent magnet synchronous motor position control system of the outer ring, finally, the whole system control effect, because the cutoff frequency of the speed loop and current loop is far less than the position loop, so in the analysis of the position loop, the assumption that there is no error current loop and speed loop, neglected, considering only the position loop, as shown in Figure 2:

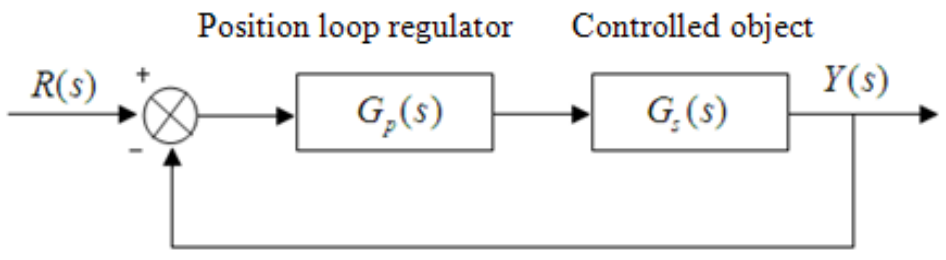

Fig.2 position loop control block diagram

The closed-loop transfer function of the system can be deduced:

$$
\Phi(s)=\frac{Y(s)}{R(s)}=\frac{G_{p}(s) G_{s}(s)}{1+G_{p}(s) G_{s}(s)}
$$

Because the gain of the closed-loop transfer function cannot be 1, there is a non - eliminating error between the actual output position and the desired position, and the gain is too large, which makes the system stability worse.

\section{Position controller design of permanent magnet synchronous motor based on iterative algorithm}

According to the characteristics of the permanent magnet synchronous motor nonlinear and strong coupling, this study uses the iterative algorithm as the position loop control method to meet the actual output of the ideal input tracking effect.

Iterative principle. Iterative algorithm is mainly used with repeating or periodic motion of the controlled object, according to the measured error signal, constantly revised to control the amount of the use of certain learning rule, in order to find an ideal input control signal, the controlled object of the output with the desired input match. The iterative control rule can be expressed as [9]:

$$
u_{k+1}(t)=u_{k}(t)+\lambda e_{k+1}(t)
$$

Where:

$k$-Iteration number; $\lambda$-Learning gain.

Controller design. The position control system adopts three closed loop structure, in order to meet the system response fast, high precision and has the advantages of simple structure, this study adopts iterative algorithm combined with PID control and control method, as shown in Figure 3.

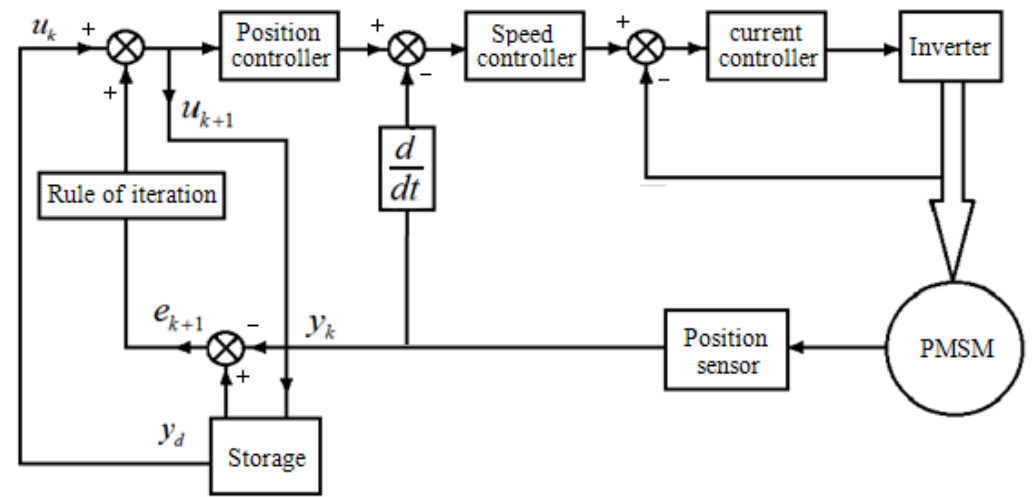

Fig.3 position control system structure of permanent magnet synchronous motor based on iterative algorithm

Figure, memory for storing a target value, and on an iterative process of actual output value, system runs for the first time, iterative learning controller is not involved in the control process, the 
whole system by PID control operation. After each run, the memory is stored in the output value $u_{k}$, after the last execution process, and the actual output is compared with the target value phase to get the error signal $e_{k}$.

\section{Simulation analysis}

In order to verify the effectiveness of the design control method, the system simulation analysis is carried out, and system simulation model is built in the MATLAB software, as shown in Figure 4.

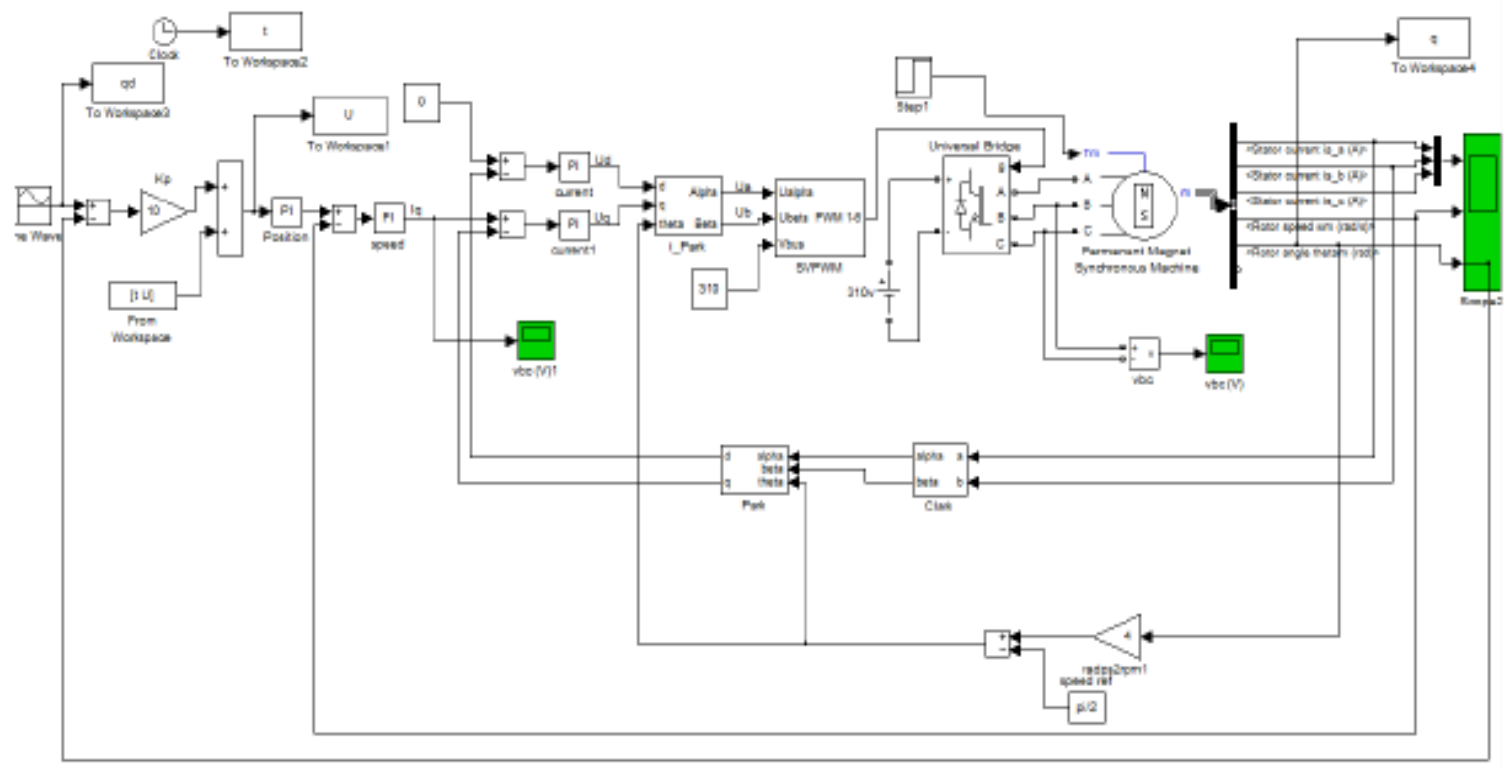

Fig.4 simulation model of position control for permanent magnet synchronous motor

In the simulation, the motor parameters are as follows: Stator resistance is $0.35 \Omega$, permanent magnet flux linkage is $0.09 \mathrm{~Wb}$, Moment of inertia is $5 \times 10^{-4} \mathrm{~kg} \cdot \mathrm{m}^{2}$, Number of pole-pairs is 4 , and friction coefficient is 0 . Simulation results are shown in Figure 5-8.

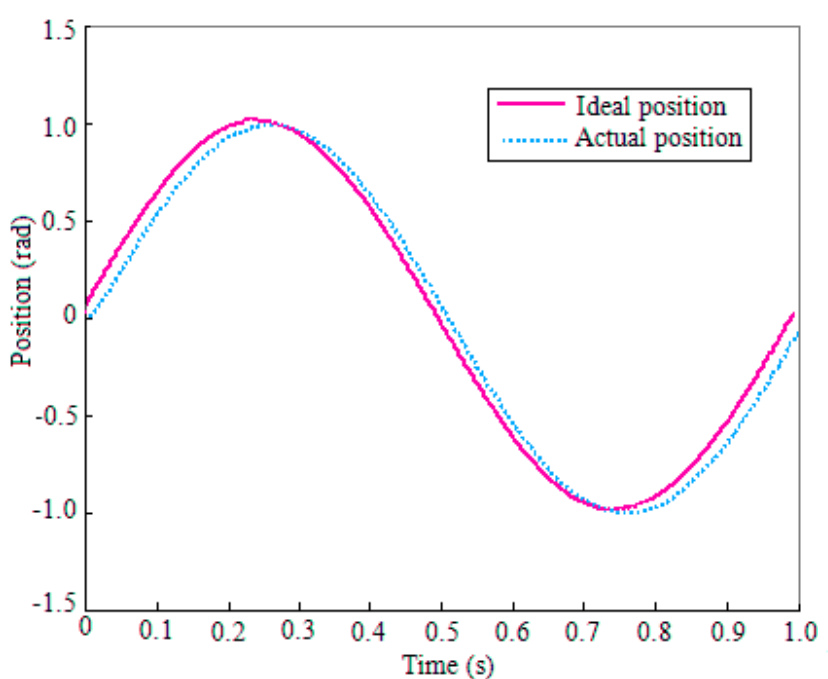

Fig.5 position curve when the first iteration

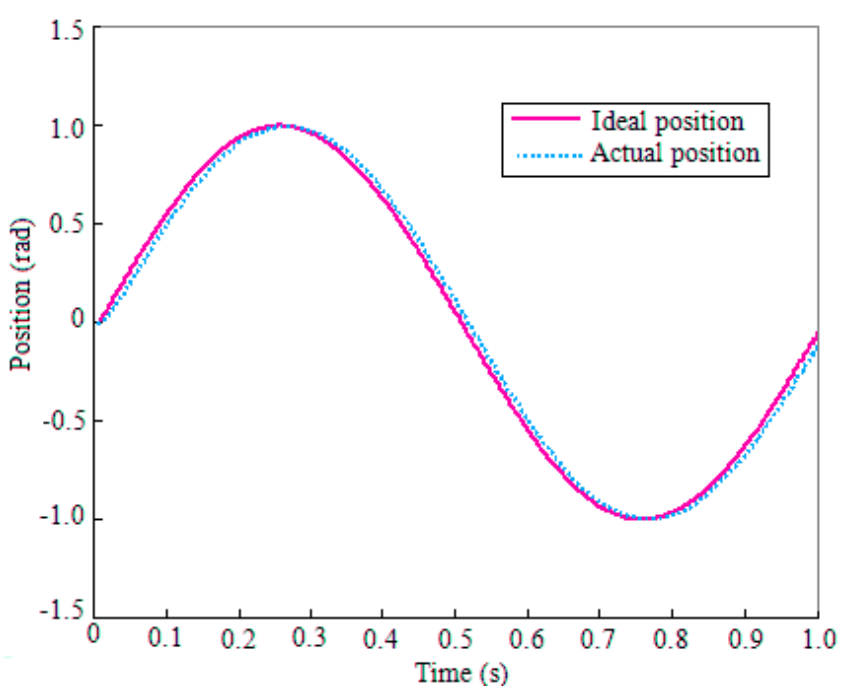

Fig.6 position curve when the third iteration 


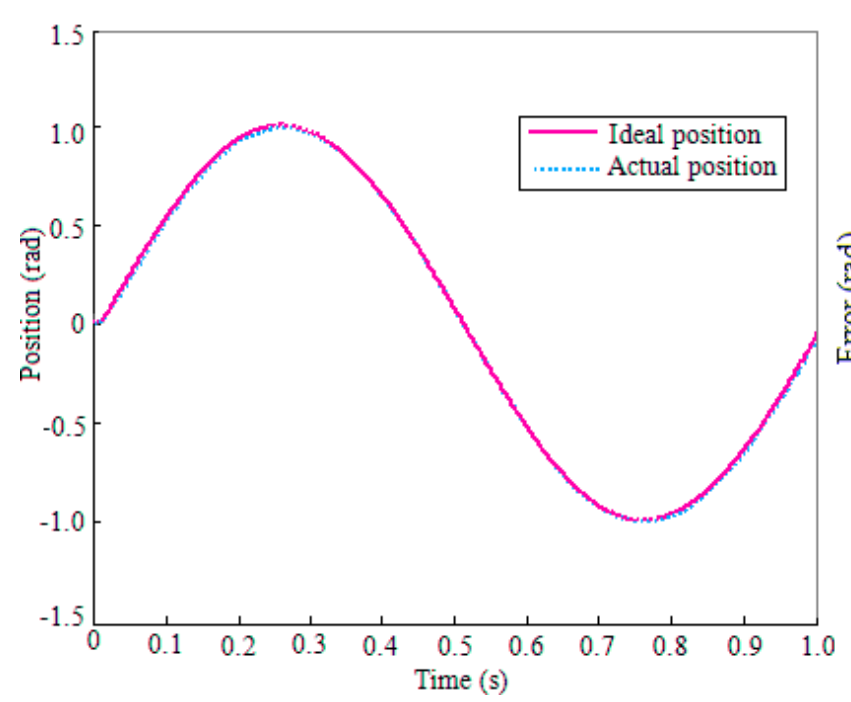

Fig.7 position curve when the fifth iteration

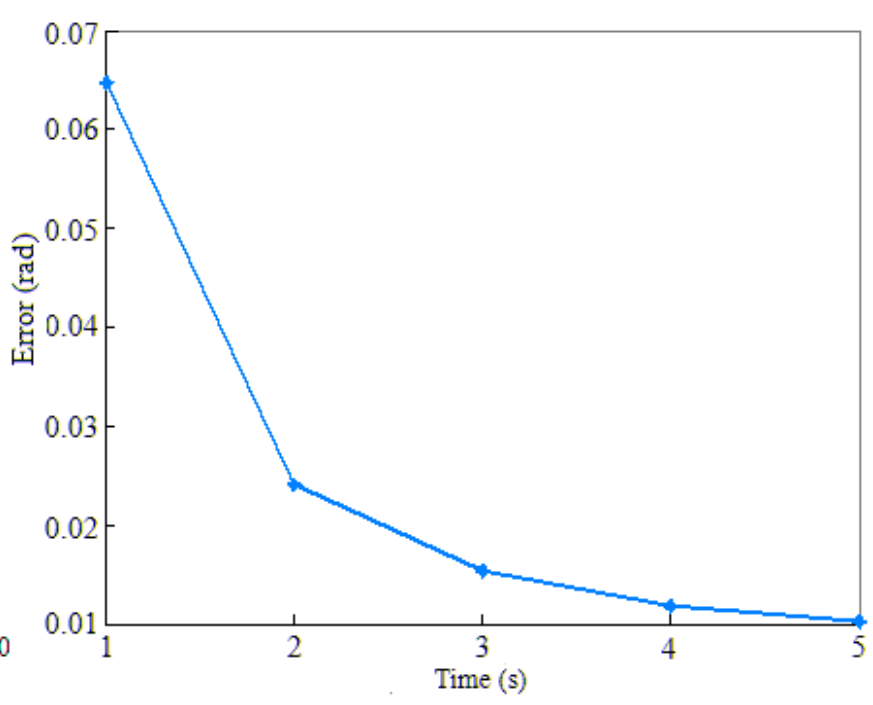

Fig.8 absolute value of position error

Simulation results show that when the first iteration, system of real output and the desired target also exist large errors, with the increase in the number of iterations, the difference between the two decreased gradually, more and more close to the target value, the so as to verify the validity of the method.

In order to solve the problem of permanent magnet synchronous motor magnetic pole position is difficult to measure the established mathematical model of permanent magnet synchronous motor (PMSM), find out the factors that affect the dynamic characteristics of, control strategy based on the position of the iterative algorithm is proposed. MATLAB simulation results show that with the increase of the number of iterations, the actual output of the system gradually expectations, which verifies the feasibility of the theoretical analysis is correct and the iterative algorithm applied in synchronous motor position control.

\section{References}

[1] Sadegh Vaez-Zadeh.Variable flux control of permanent magnet synchronous motor drives for constant torque operation [J]. IEEE Transactions on Power Electronics, 2001, 16(4) :527-534

[2] Zheng J F, Feng Y, Lu Q L. High-order terminal sliding-mode control for permanent magnet synchronous motor[J]. Control Theory \& Applications, 2009, 26(6): 697-700.

[3] Tae-Suk Kwon, seung-Ki Sul. Novel Antiwindup of a Current Regulator of a Surface-Mounted Permanent-Magnet Motor for Flux-Weakening Control [J]. IEEE Transaction on Industry Application, 2006, 42(5):1293-1300.

[4] Yang S S, Zhong Y S. Robust controller design for PMSM speed servo systems [J]. Proceedings of CSEE, 2009, 39(3): 84-90.

[5] Marco Tursini, Chiricozzi. Surface Mounted Permanent Magnet Synchronous Motors Accounting for Resistive Voltage Drop [J]. IEEE Trans on Industry Electronics, 2010, 57(1):440-448.

[6] Lu Wenqi, Hu Yuwen, et al. Anti-disturbance adaptive control for permanent magnet synchronous motor servo system[J]. Proceedings of the CSEE, 2011，31(3):75-81.

[7] Yao Wenxi, Lv Zhengzi, Fei Wanmin. The Implement Method of Three-level Inverter SVPWM with DSP Based on PWM Analytical [J]. The Power System and Automation, 2004, 28 (14): 50-54.

[8] Shinn-Ming Sue, Ching Tsai Pan. Voltage Constraint Tracking Based Field Weakening Control of IPM Synchronous Motor Drives [J].IEEE Transaction on Industry Application, 2008, 55(1):340-347. 